\title{
Platypnea orthodeoxia syndrome in a patient with pulmonary arterial hypertension
}

\author{
T Sinanis*, U Krüger, and WH Schöls \\ Department of Cardiology, Heart Center Duisburg, Germany
}

\begin{abstract}
Platypnea orthodeoxia syndrome (POS) is a rare syndrome traditionally defined as dyspnea or hypoxemia observed when changing from a recumbent to an upright or seated position. A patent foramen ovale (PFO) is oft part of the underlying pathophysiology. Here, a 70-year-old woman with known PFO presents with new symptoms of a platypnea orthodeoxia syndrome. A pulmonary arterial hypertension (PAH), treated with Ambrisentan for years, was also known. In order to clarify the etiology of the new hemodynamic state we performed a transesophageal echocardiography (TEE) in an upright position, which excluded a PFO related cause of symptoms. A further investigation shown a COPD (Chronic Obstructive Pulmonary Disease), the treatment of which relieved of symptoms. This is the first described case of Platypnea orthodeoxia syndrome in patient with PAH. Although PFO is a common cause of POS and a PFO closure is often advised, careful differential diagnosis should be kept in mind.
\end{abstract}

\section{Introduction}

Dyspnea is a frequently encountered symptom in medicine. It is one of the main reasons of presentation in the emergency department and one of the most common causes of hospitalization [1]. Dyspnea is also a complaint of nearly $4 \%$ of patients seeking treatment in the ambulatory care setting, and population samples have determined the prevalence of dyspnea to be between $17 \%$ and $38 \%$ [2]. Platypnea orthodeoxia syndrome (POS) is traditionally defined as dyspnea or hypoxemia observed when changing from a recumbent to an upright or seated position [3]. Although there are several potential causes for this phenomenon, POS is classically described as resulting from right-toleft shunting through a patent foramen ovale (PFO) [4,5]. The precise mechanism of shunting through a PFO despite normal intracardiac pressures, however, remains obscure [6].

\section{Case report}

A 70-year old woman was referred to our hospital for further investigation of deteriorating shortness of breath. Her symptoms were present for the last 3 months and were felt to have slowly worsened over time.

The patient had been diagnosed with pulmonary arterial hypertension (PAH) in 2010. Right heart catheterization at that time revealed an elevated mean pulmonary artery pressure of $47 \mathrm{~mm} \mathrm{Hg}$ and a normal wedge pressure (PAWP or PC) of $12 \mathrm{~mm} \mathrm{Hg}$. Cardiac index was reduced $\left(1.77 \mathrm{~L} / \mathrm{min} / \mathrm{m}^{2}\right)$ and pulmonary vascular resistance was significantly elevated (12 Wood units). A rheumatic arthritis with symmetric involvement of multiple joints and positive CCP-antibodies was also known since 2009. For treatment of her arthritis the patient received methotrexat once a week. As no other etiology for PAH was found, the patient's disease was classified as Dana Point group 1. She was referred to our PAH clinic for specific therapy. Sildenafil was not tolerated and bosentan lead to a drastic elevation of GOT and GPT. Ambrisentan, on the other hand, was well tolerated, improved 6 minute walking distance by 35 meters (from 180 meters to 215 meters) and decreased NT-proBNP plasma levels. Ambrisentan monotherapy was continued through the years 2010 to 2013 as the patient's general condition stabilized and dyspnea was reduced.

As a result of the $\mathrm{PAH}$, the right ventricle dilated and the right ventricular systolic function declined. With $17 \mathrm{~mm}$, TAPSE was within the normal range. Regarding echocardiographic parameters, left ventricular systolic and diastolic function showed no abnormalities.

At presentation, the patient revealed no alarming clinical signs: RR was 100/60 $\mathrm{mm} \mathrm{Hg}$, temperature $36.8^{\circ} \mathrm{C}$, blood glucose $106 \mathrm{mg} / \mathrm{dl}$. On blood gas analysis pO2 was found to be $59 \mathrm{~mm} \mathrm{Hg}$, pCO2 $43 \mathrm{~mm}$ $\mathrm{Hg}, \mathrm{BE}+6 \mathrm{mmol} / \mathrm{l}, \mathrm{pH} 7,45$, and $\mathrm{sO} 290 \%$. Blood chemistry provided the following parameters: troponin 15,6 pg/ml, NT-proBNP $126 \mathrm{pg} /$ $\mathrm{ml}$, hemoglobin $11,3 \mathrm{~g} / \mathrm{dl}$, creatinine $0,5 \mathrm{mg} / \mathrm{dl}$, potassium $3,5 \mathrm{mmol} / \mathrm{l}$, sodium $140 \mathrm{mmol} / \mathrm{l}, \mathrm{CRP}<0,5 \mathrm{mg} / \mathrm{dl}$. The electrocardiogram showed a sinus rhythm ( 95 beats pro minute) with a known right axis and peaked $\mathrm{P}$ waves as a result of the pulmonary hypertension (Figure 1A). The chest X-ray had no signs of pulmoary congestion, pleural effusion or pneumonia (Figure 1B).

The patient described increasing shortness of breath while standing with an improvement when changing to a recumbent position. To substantiate this perception, continuous measurements of heart rate and oxygen saturation in lying, sitting and standing positions were obtained. Results are summarized in Table 1.

For further investigation, transesophageal echocardiography (TEE) was performed, combined with continuous registration of heart rate and oxygen saturation. Agitated saline contrast was used to search for intracardiac shunts (Figure 2). As a significant right-to-left shunt would increase left atrial pressure and reduce pulmonary venous flow,

Correspondence to: Theodoros Sinanis, Department of Cardiology, Heart Center Duisburg, Germany, E-mail: tsinanis@web.de

Received: December 03, 2017; Accepted: December 21, 2017; Published: December 23, 2017 


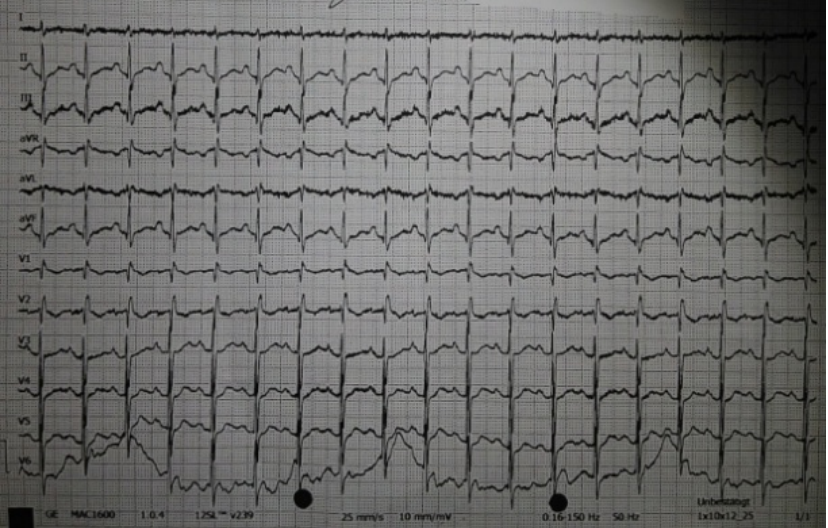

A

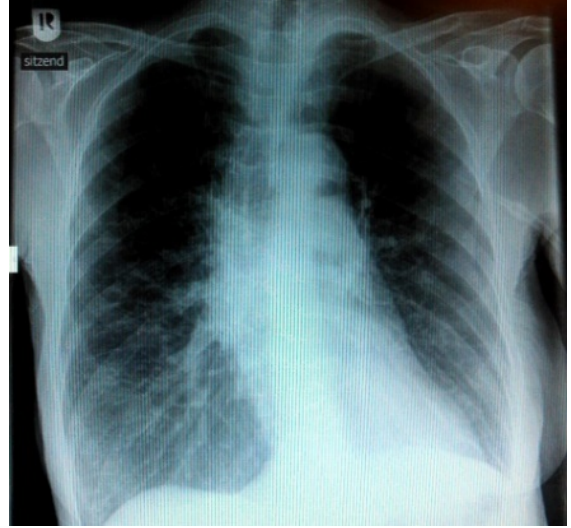

B

Figure 1. 12 lead electrocardiogram (A) and chest X-ray (B) of the patient
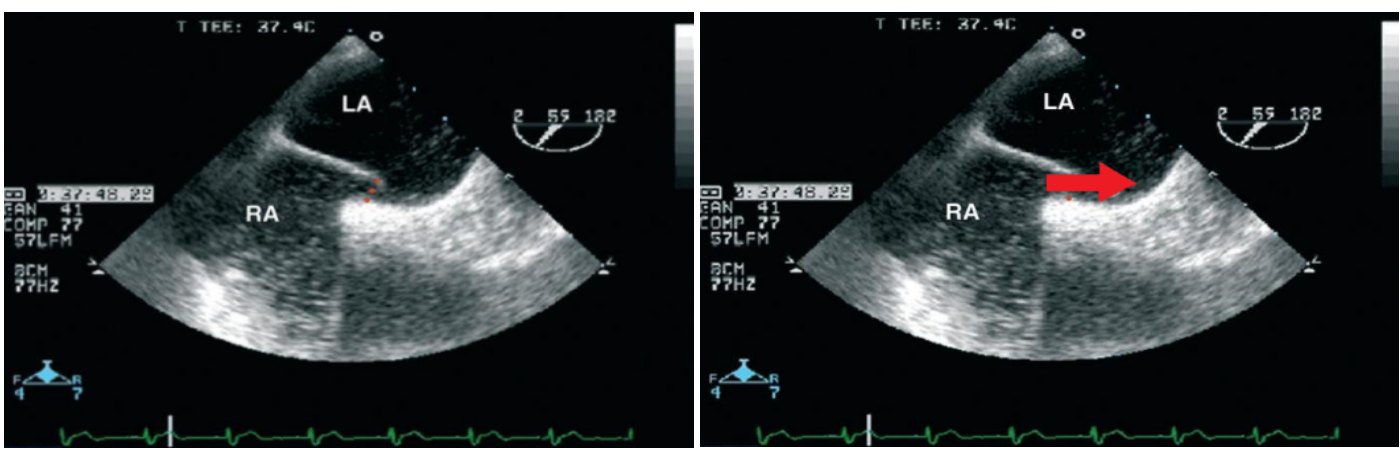

Figure 2. Presentation of the PFO without evidence of obstruction to the flow in the pulmonary veins.

Table 1. Changes in oxygen saturation $(\mathrm{SO} 2)$ and in heart rate (HF) in relation to the position of the patient. $\mathrm{bpm}=$ beats per minute.

\begin{tabular}{|c|c|c|c|c|c|c|}
\hline minutes & \multicolumn{2}{|c}{ lying } & \multicolumn{2}{c|}{ sitting } & \multicolumn{2}{c|}{ standing } \\
\hline & SO2 (\%) & HF (bpm) & SO2 (\%) & HF (bpm) & SO2 (\%) & HF (bpm) \\
\hline $\mathbf{0}$ & 89 & 110 & 89 & 126 & 85 & 132 \\
\hline $\mathbf{1}^{\prime}$ & 89 & 110 & 87 & 120 & 83 & 133 \\
\hline $\mathbf{3}^{\prime}$ & 89 & 110 & 86 & 123 & 82 & 135 \\
\hline $\mathbf{5}^{\prime}$ & 89 & 110 & 85 & 123 & 82 & 134 \\
\hline $\mathbf{1 0}^{\prime}$ & 89 & 110 & 84 & 121 & 81 & 137 \\
\hline
\end{tabular}

the latter was also evaluated by Doppler echocardiography. The goal was to elucidate the potential role of the previously diagnosed persistent foramen ovale (PFO) in the pathophysiology of the clinical symptoms.

In lying position, there was no relevant transition of $\mathrm{NaCL}$ bubbles from the right to the left atrium. Accordingly, the flow in the pulmonary veins was not compromised. This was in line with the fact that oxygen saturation reached $89 \%$ in lying position, compatible with the patient's pulmonary hypertension and not suspicious of any right-to-left shunt. When moving the patient to an upright position with the TEE probe in place, the oxygen saturation dropped to $81 \%$. Agitated saline contrast was applied once again, but failed to provide any evidence of intracardiac shunting. Furthermore, pulmonary venous flow was unchanged under these conditions as well. Contrary to our expectations, the PFO did not seem to account for the platypnoe orthodeoxia syndrome. Thus, the diagnostic work-up was extended. Pulmonary function testing revealed chronic obstructive pulmonary disease. Subsequently, inhalative antiinflammatoric/antiobstructive treatment and non-invasive ventilation (NIV) therapy was initiated. Within 2 weeks, the patient's symptoms resolved.

\section{Discussion}

Platypnea-orthodeoxia syndrome (POS) is an uncommon condition characterized by dyspnea and arterial desaturation upon assuming an upright position [7,8]. The syndrome was originally described in patients with pulmonary disease without intracardiac shunts [8].

Current knowledge relates POS to intracardiac shunting, intrapulmonary shunting, ventilation-perfusion mismatch, or a combination of these $[9,10]$.

In the case of anatomical interatrial discontinuities (e.g., patent foramen ovale, atrial septal defects), a functional component modified by positional changes is required as well. Functional components include conditions that increase right atrial pressure, stretch the interatrial septum and/or redirect blood flow through the interatrial communication.

Anatomical as well as functional conditions may lead to intrapulmonary shunting. Arteriovenous anastomoses, pulmonary arteriovenous malformations and grossly distended pulmonary capillaries are potential causes of anatomical shunts. Functional shunts are due to ventilation-perfusion mismatch, that is, hypoventilation of normally perfused segments or hyperperfusion of normally ventilated segments. In either case, insufficiently oxygenated blood mixes with oxygenated blood, thereby resulting in overall desaturation. The mechanisms affecting the degree of intrapulmonary shunting and their relation to positional changes are not fully understood. Clearly, in the upright position, pulmonary perfusion shifts from the apical to the basal parts of the lungs. Right ventricular preload is reduced, and 
pulmonary artery pressure drops. The effect on pulmonary venous pressure is less predictable, considering the plethora of modifying factors, such as left ventricular function and compliance, left ventricular pre- and afterload, heart rate valvular function. Irrespective of other potential mechanisms, the apico-basal shift of pulmonary perfusion upon standing aggravates the functional significance of any basally located pathology [11]. More pronounced anatomical or functional intrapulmonary shunting in the lower parts combined with a reduced potential for compensation in the upper parts of the lungs will then result in significant deoxygenation. In line with these considerations, our patient also revealed a basal predominance with respect to his COPD (Chronic Obstructive Pulmonary Disease).

The diagnosis of POS is based on the following criteria: 1) platypnea, 2) orthodeoxia (SpO2 <90\%), 3) existence of an interatrial or intrapulmonal communication, and 4) right-to-left shunt. Our patient presented with typical symptoms, and the previously diagnosed PFO seemed to provide the pathophysiologic basis. However, contrary to expectations, we were unable to demonstrate a right-to-left shunt across this interatrial discontinuity. As described by Desouza [12], tilt TEE with agitated saline contrast solution is the most effective diagnostic tool for this purpose. In rare cases, a shunt is only seen with Valsalva maneuver, which could not performed in our patient because of his apparent shortness of breath. However, if this shortness of breath would have been related to an interatrial right-to-left shunt, it would have had to be present under just these conditions. In the absence of an intracardiac shunt, contrast-enhanced echocardiography, perfusion scan (scintigraphy) with macroaggregated albumin or pulmonary arteriography might be used to detect intrapulmonary shunting [13]. Based on the favourable clinical course, we refrained from respective diagnostic procedures in our patient.

If POS is definitely related to intracardiac shunting, closure of the interatrial communication represents definitive treatment. In 2001, Rao et al. [14] reported on percutaneous closure of atrial septal defects and PFOs in platypnea-orthodeoxia patients via femoral approach. A jugular approach is feasible as well [15]. Transcatheter closure immediately improves oxygen saturation in upright position. Platypnea resolves completely [16-18]. Symptomatic improvement is seen in $>95 \%$ of patients. In light of this effective treatment option, it is tempting to proceed with respective interventions once an interatrial communication has been detected. However, this case clearly demonstrates that a PFO might be an "innocent bystander", where closure would not affect the underlying pathophysiology. Instead, particularly in patients with pulmonary hypertension and right heart failure, an intervention could even be detrimental by preventing preload reduction through right-to-left shunting.

In patients with intrapulmonary shunting, the underlying pulmonary disease must be treated to reduce ventilation-perfusion mismatch. Closure of small airways or microatelectasis have been suggested as potential causes of ventilation-perfusion mismatch in platypnoea-orthodeoxia [19], possibly reversible with CPAP ventilation. Both, mechanical ventilation and application of endexpiratory positive airway pressure (PEEP) tend to increase right-toleft shunting with worsening of hypoxaemia. Our patient experienced a gradual improvement in oxygenation while on mechanical ventilation with PEEP application and also during non-invasive bi-level ventilation.

\section{Conclusion}

Awareness and careful clinical evaluation are clues to the diagnosis of POS. Although an interatrial communication is frequently involved in the underlying pathophysiology, it might also coexist without any causal relationship. Thus, a functional analysis of all potential components is required before deciding on a specific therapy.

\section{References}

1. Olsson T, Terent A, Lind L (2004) Rapid Emergency Medicine Score can predict longterm mortality in nonsurgical emergency department patients. Acad Emerg Med 11: 1008-13. [Crossref]

2. Sarkar S, Amelung PJ (2006) Evaluation of the dyspneic patient in the office. Prim Care 33: 643-657. [CrossRef]

3. Toffart AC, Bouvaist H, Feral V, Blin D, Pison C (2008) Hypoxemia- orthodeoxia related to patent foramen ovale without pulmonary hypertension. Heart Lung 37: 3859. [Crossref]

4. Guérin P, Lambert V, Godart F, Legendre A, Petit J, et al. (2005) Transcatheter closure of patent foramen ovale in patients with platypnea-orthodeoxia: results of a multicentric French registry. Cardiovasc Intervent Radiol 28: 164-8. [Crossref]

5. Delgado G, Inglessis I, Martin-Herrero F, Yoerger D, Liberthson R, et al. (2004) Management of platypnea-orthodeoxia syndrome by transcatheter closure of atria communication: hemodynamic characteristics, clinical and echocardiographic outcome. J Invasive Cardiol 16: 578-82. [Crossref]

6. Zanchetta M, Rigatelli G, Ho SY (2005) A mystery featuring right-to-left shunting despite normal intracardiac pressure. Chest 128: 998-1002. [Crossref]

7. Seward JB, Hayes DL, Smith HC, Williams DE, Rosenow EC 3rd, et al. (1984) Platypnea-orthodeoxia: clinical profile, diagnostic workup, management, and report of seven cases. Mayo Clin Proc 59: 221-31. [Crossref]

8. Robin ED, McCauley RF (1997) An analysis of platypnea-orthodeoxia syndrome including a "new" therapeutic approach. Chest 112: 1449-51. [Crossref]

9. Chen GP, Goldberg SL, Gill EA Jr (2005) Patent foramen ovale and the platypneaorthodeoxia syndrome. Cardiol Clin 23: 85-9.

10. Blanche C, Noble S, Roffi M, Testuz A, Muller H, et al. (2013) Platypnea-orthodeoxia syndrome in the elderly treated by percutaneous patent foramen ovale closure: a case series and literature review. Eur J Intern Med 24: 813-7. [Crossref]

11. Newton PN, Wakefield AE, Goldin R, Govan J (2003) Pneumocystis carinii pneumonia with pleurisy, platypnoea and orthodeoxia. Thorax 58: 185-186. [Crossref]

12. Desouza KA1, Saraswat S, DeSouza SA, Rajaram V, Reddy PC, et al. (2009) Platypneaorthodeoxia syndrome: a diagnostic challenge. South Med J 102: 1046-1048. [Crossref]

13. Lee CH, Cheng ST (2011) Shortness of breath while sitting up: hepatopulmonary syndrome. Can Med Ass J 183: 80. [Crossref]

14. Burchell HB, Helmholz HF Jr Wood EH (1949) Reflex orthostatic dyspnea associated with pulmonary hypertension. Am J Physiol 159: 563-4.

15. Godart F, Rey C (2001) Platypnea Orthodeoxia Syndrome: A probably underestimated syndrome? Chest 5: 1624-1625. [Crossref]

16. Adolph EA, Lacy WO, Hermoni YI, Wexler LF, Javaheri S (1992) Reversible orthodeoxia and platypnea due to right-to-left intracardiac shunting related to pericardial effusion. Ann Intern Med 116: 138-139. [Crossref]

17. Hirai N, Fukunaga T, Kawano H, Honda O, Sakamoto T, et al. (2003) PlatypneaOrthodeoxia Syndrome with atrial septal defect. Circ J 67: 172-175. [Crossref]

18. Nakahira A, Matsumura Y, Tatsumi H, Sasaki Y, Hirai H, et al. (2010) Platypneaorthodeoxia diagnosed by sitting transesophageal echocardiography. Ann Thorac Surg 89: 1284-1286. [Crossref]

19. Fox JL, Brown E, Harrison JK, Williams J, Terry PB (1989) Platypnea-orthodeoxia and progressive autonomic failure. Am Rev Respir Dis 140: 1802-4. [Crossref]

Copyright: (C)2017 Sinanis T. This is an open-access article distributed under the terms of the Creative Commons Attribution License, which permits unrestricted use, distribution, and reproduction in any medium, provided the original author and source are credited. 